\title{
Catching Common Cold Virus with a Net: Pyridostatin Forms Filaments in Tris Buffer That Trap Viruses-A Novel Antiviral Strategy?
}

\author{
Antonio Real-Hohn ${ }^{1, *}$, Rong Zhu ${ }^{2}$, Haleh Ganjian ${ }^{3}$, Nahla Ibrahim ${ }^{4}\left(\mathbb{D}\right.$, Peter Hinterdorfer ${ }^{2}$, \\ Heinrich Kowalski ${ }^{1}$ and Dieter Blaas ${ }^{1}$ \\ 1 Vienna Biocentre, Max Perutz Laboratories, Medical University of Vienna, Center of Med. Biochemistry, \\ Dr. Bohr Gasse 9/3, A-1030 Vienna, Austria; heinrich.kowalski@meduniwien.ac.at (H.K.); \\ dieter.blaas@meduniwien.ac.at (D.B.) \\ 2 Institute of Biophysics, Johannes Kepler University Linz, Gruberstr. 40, A-4020 Linz, Austria; \\ rong.zhu@jku.at (R.Z.); peter.hinterdorfer@jku.at (P.H.) \\ 3 Department of Laboratory Medicine, Division of Clinical Microbiology, Karolinska Institutet, Alfred Nobels \\ Allé 8/7, 14152 Huddinge, Sweden; haleh.ganjian@temple.edu \\ 4 Department of Surgery, Surgical Research Laboratory, Medical University of Vienna, Waehringer Guertel \\ 18-20, A-1090 Vienna, Austria; nahla.ibrahim@nyu.edu \\ * Correspondence: antonio.hohn@meduniwien.ac.at; Tel.: +43-1-4277-61631
}

Received: 15 May 2020; Accepted: 30 June 2020; Published: 4 July 2020

check for updates

\begin{abstract}
The neutrophil extracellular trap (ET) is a eukaryotic host defense machinery that operates by capturing and concentrating pathogens in a filamentous network manufactured by neutrophils and made of DNA, histones, and many other components. Respiratory virus-induced ETs are involved in tissue damage and impairment of the alveolar-capillary barrier, but they also aid in fending off infection. We found that the small organic compound pyridostatin (PDS) forms somewhat similar fibrillary structures in Tris buffer in a concentration-dependent manner. Common cold viruses promote this process and become entrapped in the network, decreasing their infectivity by about $70 \%$ in tissue culture. We propose studying this novel mechanism of virus inhibition for its utility in preventing viral infection.
\end{abstract}

Keywords: Enterovirus; Rhinovirus; pyridostatin; filament; extracellular traps

Text

Extracellular traps (ETs) are fibrillary networks formed from $\sim 16 \mathrm{~nm}$ diameter filaments constituted mainly of nuclear or mitochondrial DNA from mast cells, eosinophils, macrophages, and neutrophils [1]. ETs carry histones, gelatinase, proteinases, elastase, cathepsins, lactoferrin, myeloperoxidase, and other defensins and are part of the innate immune system [2]. ET production is triggered by bacteria, but more recently, viruses were found to also induce ETs [3]. For example, influenza virus infection gives rise to specific ETs that cannot cross-neutralize bacteria but generate inflammation that undermines the alveolar-capillary barrier function and thereby promotes secondary bacterial infection. However, neutrophil ETs also inhibit virus infection [4]. We here demonstrate, via immunolabeling, that a rhinovirus interacts with ETs (Figure S1 and Movie S1). Viruses cannot escape from ETs, as this would require nucleic acid hydrolysing enzymes at their surfaces, as are present, for example, in group A Streptococcus [5].

A recent in silico study of all available human virus genomes revealed the presence of multiple G-quadruplexes (G4s), repeats of Hoogsteen paired guanosines, in all genomes [6], including those of rhinoviruses, the main cause of the common cold. This prompted us to study whether 
G4-stabilizing compounds, such as pyridostatin (4-(2-aminoethoxy)-N2,N6-bis[4-(2-aminoethoxy)-2quinolinyl]-2,6-pyridinedicarboxamide; PDS), might interfere with the release of the ssRNA genome from a prototype rhinovirus, RV-A2, and thus prevent infection via impeding G4 unfolding. PDS and similar compounds are being investigated as anticancer drugs, as they stabilize G4s in telomeres, impacting cellular DNA replication [7]. We found that RV-A2 infection was indeed inhibited upon preincubation with PDS at room temperature. For control purposes, the same incubations of virus and PDS were also carried out at $4{ }^{\circ} \mathrm{C}$, a temperature that very much reduces the diffusion of the compound through the viral protein shell to attain the RNA; capsid breathing dynamically opens conduits in the viral capsid, but this phenomenon is highly temperature-dependent [8]. We were intrigued to see that co-incubation at $4{ }^{\circ} \mathrm{C}$ also inhibited infection, but only in Tris buffers. Inhibition of infection upon incubation at room temperature was, however, independent from the buffer and only due to the above G4-stabilization (manuscript under submission). Since PDS contains planar rings (Figure 1a), we wondered whether individual molecules might stack on top of each other and act via aggregating the virus. Such aggregates might bind the virus and reduce its effective concentration. To reveal a putative higher-order structure at an ultrastructural level, $4 \mu \mathrm{M}$ PDS in water was applied onto freshly cleaved mica and left for $5 \mathrm{~min}$. The PDS solution was then replaced with phosphate-buffered saline (PBS). Using an atomic force microscope (AFM) equipped with a fluidic cell in a Pico-SPM (Molecular Imaging, Phoenix, AZ, USA) [9], we saw that the PDS molecules attached to the mica and aggregated into long fibers with a height of $1.8 \pm 0.2 \mathrm{~nm}$ (Figure 1b). Such fibers did not form when the PDS was dissolved in PBS (Figure 1c).

The above observations pointed to a dependence of the fiber-generation on the buffer components. To investigate this, we dissolved PDS at $200 \mu \mathrm{M}$ in different buffers and applied the solutions onto glow-discharged carbon-coated electron microscopy (EM) grids, subjected them to negative staining with phosphotungstate, and observed the samples in a FEI Morgagni 268D electron microscope at $80 \mathrm{kV}$ (Figure 1d). In contrast to the sample adsorbed to mica and observed with AFM, PDS only formed small amorphous adducts in water and very few aggregates (white arrowheads) on the carbon-coated grids. 'Protofibrils'(black arrows) were also seen with PDS dissolved in Dulbecco's modified Eagle medium (DMEM, Sigma Aldrich; St. Louis, MO, USA) supplemented with 10\% fetal bovine serum (Gibco; Thermo Fisher Scientific, Waltham, MA, USA). However, fibers formed in $50 \mathrm{mM} \mathrm{NaCl}, 25 \mathrm{mM}$ Tris- $\mathrm{HCl}$ ( $\mathrm{pH} 7.5)$, but not in PBS.

To further study the PDS fiber-generating conditions, we dissolved PDS at various concentrations in the above Tris buffer and observed the samples by TEM (Figure 1e) as above. We noticed a clear concentration dependence of fiber-generation with no fibers occurring at or below $20 \mu \mathrm{M}$ PDS. 'Protofibrils' appeared at $40 \mu \mathrm{M}$ and well-defined fibers from $60 \mu \mathrm{M}$ PDS onwards.

We then investigated the influence of 20 and $100 \mu \mathrm{M}$ PDS in the above Tris buffer on the light-up of SYTO82 (Thermo Fisher Scientific), a fluorescent probe diluted to $5 \mu \mathrm{M}$ in the same buffer (Figure 1f). Nucleic acids can dramatically increase the fluorescence of such probes due to a forced planarization or rigidification of the probe [10]. For example, production of ETs by different cells has been monitored with SYTOX, a fluorescent probe that lights up upon interaction with nucleotide polymers [11]. The fluorescent signal emission intensity (excitation $541 \mathrm{~nm} /$ emission $560 \mathrm{~nm}$ ) was acquired at room temperature using a Jasco 6500 fluorometer and plotted as relative fluorescence intensity (RFU). We observed a strong increase of SYTO 82 fluorescence emission upon adding increasing concentrations of PDS. This might be taken to indicate that the PDS can arrange in higher-order structures probably due to $\pi-\pi$ stacking and a hydrophobic effect similarly observed in nucleotide polymers (reviewed in Friedman and Honig [12] and references within). 
a

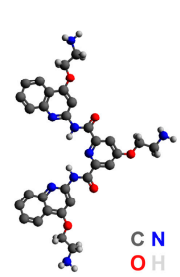

b

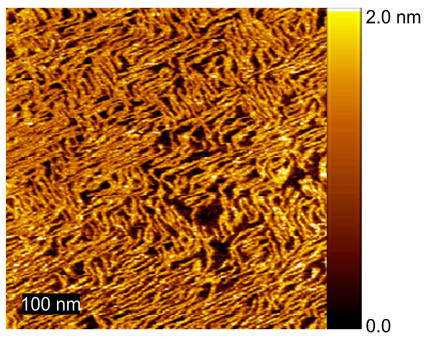

C

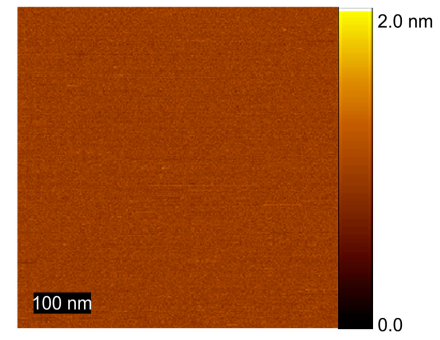

d

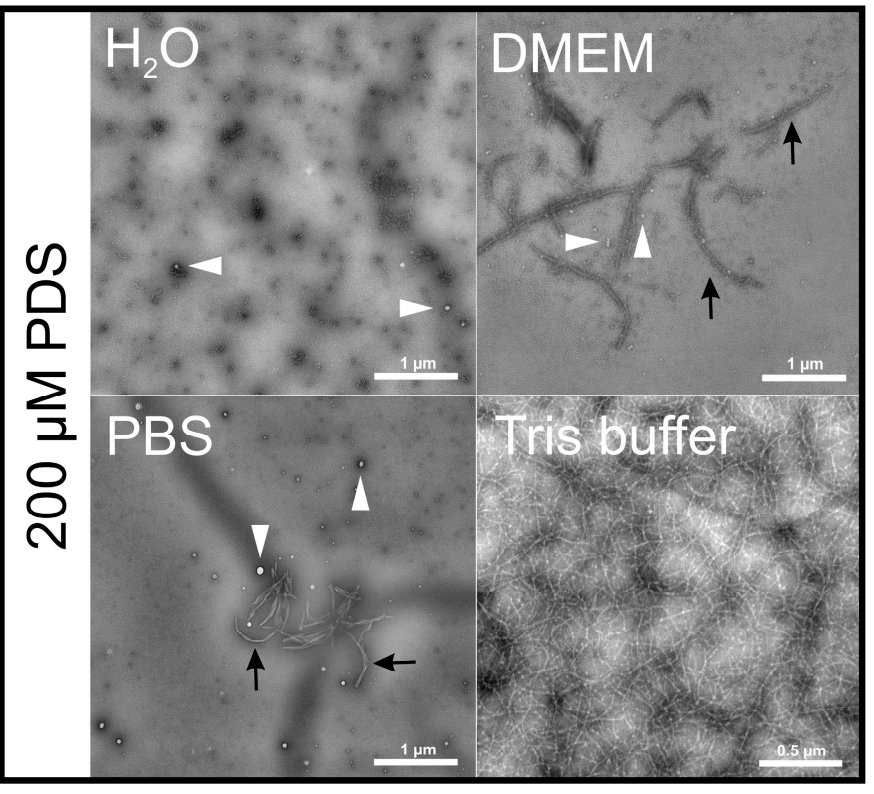

e

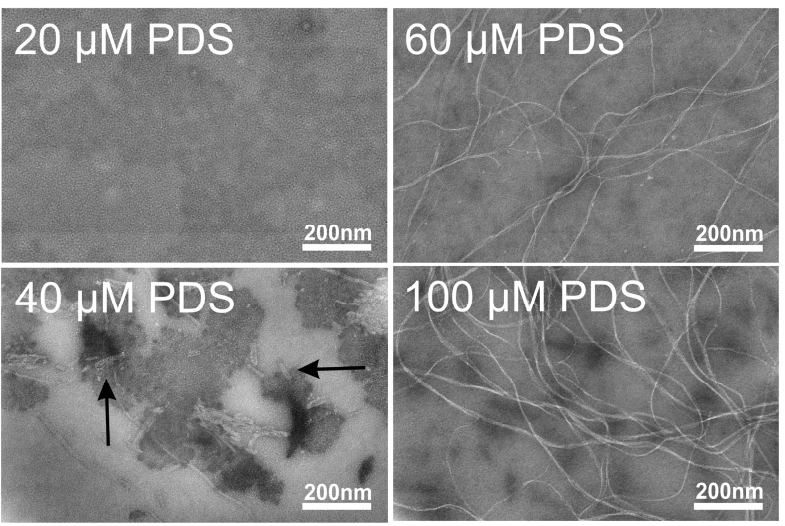

f

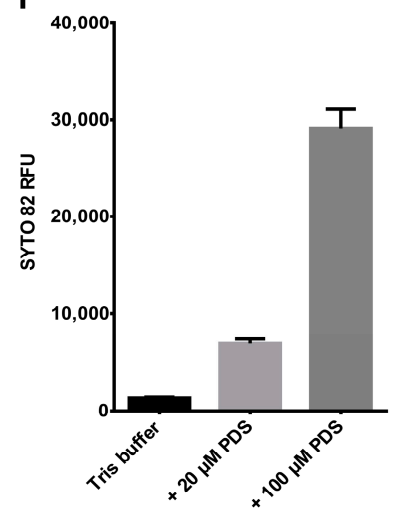

Figure 1. Formation of PDS fibrils under various conditions. (a) The structural formula of pyridostatin; for atom colour codes, see lower right. (b) PDS $(4 \mu \mathrm{M})$ in water was applied to freshly cleaved mica, incubated for $5 \mathrm{~min}$, and viewed by AFM. (c) As (b) but with $4 \mu \mathrm{M}$ PDS in PBS instead of water. (d) PDS dissolved in the solutions indicated at $200 \mu \mathrm{M}$ was applied onto EM grids, stained with $2 \%$ phosphotungstic acid ( $\mathrm{pH}$ 7.4), and imaged by TEM. The white arrowheads point to small amorphous aggregates, the black arrows to 'protofibrils'. (e) PDS dissolved in Tris buffer at the concentrations indicated was applied to an EM grid, stained with $2 \%$ phosphotungstic acid ( $\mathrm{pH} 7.4$ ), and imaged by TEM. (f) SYTO82 at $5 \mu \mathrm{M}$ in Tris buffer +/- PDS at the final concentrations indicated was transferred to a quartz cuvette and the fluorescence signal acquired at room temperature (excitation $541 \mathrm{~nm} /$ emission $560 \mathrm{~nm}$ ). Data were analysed by one-way ANOVA with Tukey's multiple comparisons, revealing that the values are significantly different from each other $(p<0.05) . n=3$. 
Taken together, the AFM and TEM observation of PDS producing networks of fibers and the increase of the SYTO82 nucleic acid binding capacity led us to ask whether the PDS fibers might be capable of trapping viruses similar to ETs and thereby inhibiting viral infectivity, despite being completely different with respect to their composition. To test that in a physiologically relevant system, we measured the infectivity of PDS-treated virus in HeLa cells. We are aware that such an experiment does not take into account that the fibers might damage the cells similarly to natural ETs (see above); if so, the decreased cell survival could be misinterpreted as increased infectivity. To avoid this, we first decreased the PDS concentrations to $20 \mu \mathrm{M}$ and incubated $1 \mu \mathrm{g} / \mathrm{mL}$ RV-A2 in Tris buffer for $30 \mathrm{~min}$ on ice to prevent capsid breathing and, thus, the interaction of PDS with the viral genome within the protein shell. TEM observation suggested that the presence of virus particles induced the formation of fibers, as they were already observed at the low concentration of $20 \mu \mathrm{M}$ PDS (Figure 2a). Note that the shape of the PDS fibers differs to some extent in different experiments (left panel).
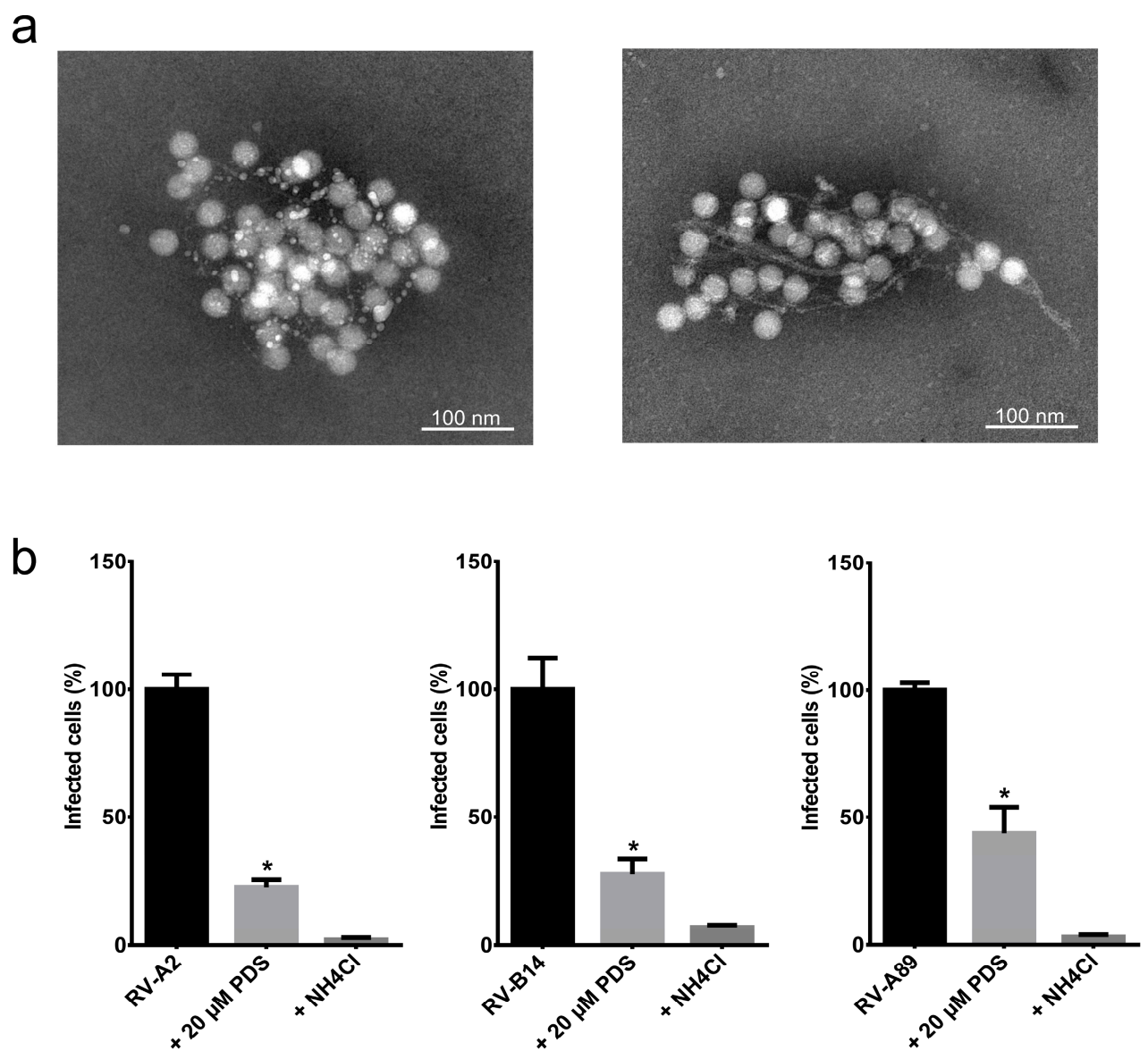

Figure 2. PDS fibrils entrap various RVs. (a) RV-A2 $(1 \mu \mathrm{g} / \mathrm{mL})$ was mixed with PDS at $20 \mu \mathrm{M}$ in Tris buffer, transferred onto EM grids, stained with $2 \%$ phosphotungstic acid ( $\mathrm{pH} 7.4$ ), and viewed by TEM. (b) HeLa cells, 80\% confluent, were either infected with RV-A2, RV-B14, or RV-A89, respectively (controls), or pre-incubated with $20 \mu \mathrm{M}$ PDS in Tris buffer for $30 \mathrm{~min}$ on ice prior to infection as above. The samples were diluted 10 times in infection medium plus $25 \mathrm{mM} \mathrm{NH}_{4} \mathrm{Cl}$ and added to the cells. One hour post-challenge, the medium was replaced with fresh infection medium without $\mathrm{NH}_{4} \mathrm{Cl}$ to initiate uncoating. As a second control, $\mathrm{NH}_{4} \mathrm{Cl}$ was maintained throughout the experiment. At $8 \mathrm{~h}$ post-infection, the cells were prepared for immunofluorescence, and the number of cells producing viral antigen, indicating infection, was determined in a TissueFAXS. The average and standard error of the mean of infected cells from three independent assays were plotted. The figure was prepared and the significance levels determined by using GraphPad Prism 6.0 using one-way ANOVA. * $p<0.0001$ vs. RVs without PDS. 
To test whether the PDS fibers would indeed trap the virus and thus reduce infection, we grew HeLa cells until sub-confluent on coverslips and challenged them with RV-A2, RV-B14, and RV-A89, respectively, at a multiplicity of infection (MOI) of 100, as in Real-Hohn et al. [13]; the viruses had been diluted in Tris buffer with $20 \mu \mathrm{M}$ PDS as above and incubated on ice for $30 \mathrm{~min}$. For control, the viruses were incubated on ice for $30 \mathrm{~min}$ in the same buffer but in the absence of PDS. The mixture was then diluted ten times with infection medium (DMEM plus $2 \%$ fetal bovine serum) and transferred onto the cells on the coverslips. The respective virus was allowed to enter the cells, but not to uncoat by the presence of $25 \mathrm{mM} \mathrm{NH}_{4} \mathrm{Cl}$ for $1 \mathrm{~h}$ at $34^{\circ} \mathrm{C}$; ammonium ions neutralize the endosomal acidic $\mathrm{pH}$ preventing the structural changes necessary for the release of viral RNA and infection. $\mathrm{NH}_{4} \mathrm{Cl}$ was washed away, setting the time for synchronized productive infection. At $8 \mathrm{~h}$ post-infection, the cells were washed, fixed, permeabilized, and blocked with goat serum. The RV-A2-infected sample was incubated with monoclonal antibody $8 \mathrm{~F} 5$, diluted to $10 \mu \mathrm{g} / \mathrm{mL}$ in goat serum, and the RV-B14 and RV-A89 infected samples with specific rabbit antisera diluted to 1:500 in goat serum. For detection, secondary antibodies labeled with Alexa fluor (Life Technologies, Carlsbad, CA, USA) were used at $1 \mu \mathrm{g} / \mathrm{mL}$, followed by extensive washing. The slides were mounted, and fluorescence microscopy images were recorded on a TissueFAXS automated microscope (TissueGnostics, Vienna, Austria) as in Ganjian et al. [14]. The presence of PDS diminished the number of virus-positive cells in all cases by about $70 \%$ compared with cells identically infected with the respective untreated virus. In the continuous presence of the uncoating inhibitor $\mathrm{NH}_{4} \mathrm{Cl}$, virus production was reduced to about $99 \%$ in each instance (Figure 2b).

Finally, we induced release of neutrophil ETs with phorbol 12-myristate 13-acetate acetate (PMA) from isolated neutrophils and observed significant capturing of added RV-A2 particles on elastase-decorated chromatin-based nets (Figure S1 and Movie S1). Taken together, we conclude that the PDS fibrils might act similarly to ETs by trapping the virus and preventing it from binding and being taken up by the cells. However, more work is required to find out whether the observed viral trapping could indeed be used in antiviral therapy. It had been shown that Tris, called 'Tromethamine' in the below citation, administered through nebulization alleviated respiratory problems resulting from cystic fibrosis [15]; adding the minimally toxic PDS to such solutions might be a way of application.

Supplementary Materials: The following are available online at http://www.mdpi.com/1999-4915/12/7/723/s1, Figure S1: Neutrophils were prepared from freshly collected blood from a healthy donor by using centrifugation on a density gradient; Movie S1: Freshly collected neutrophils from a healthy donor and purified as above by density gradient separation were placed on coverslips for attachment and maintained in HBSS +/+.

Author Contributions: Conceptualisation: A.R.-H., H.G., D.B. Methodology: A.R.-H., R.Z., H.G., N.I. Validation: A.R.-H., P.H., H.K., D.B. Formal Analysis: A.R.-H., R.Z., H.G. Investigation: A.R.-H., R.Z., H.G., N.I. Resources: P.H., H.K., D.B., N.I. Data Curation: A.R.-H., R.Z., H.G., D.B. Writing-Original Draft Preparation: A.R.-H. Writing-Review and Editing: A.R.-H., H.K., D.B. Visualisation: A.R.-H. Supervision: P.H., H.K., D.B. Project Administration: A.R.-H., H.K., D.B. Funding: P.H., H.K., D.B. All authors have read and agreed to the published version of the manuscript.

Funding: This research and open access were funded by the Austrian Science Fund (FWF), grant number P31392 (D. Blaas), P27196 (H. Kowalski), and P31599-B27 (R. Zhu, P. Hinterdorfer).

Acknowledgments: We thank Irene Goesler for virus growth and purification and Nadine Löffler for technical assistance.

Conflicts of Interest: The authors declare no conflicts of interest.

\section{References}

1. Goldmann, O.; Medina, E. The expanding world of extracellular traps: Not only neutrophils but much more. Front. Immunol. 2012, 3, 420. [CrossRef] [PubMed]

2. Brinkmann, V.; Reichard, U.; Goosmann, C.; Fauler, B.; Uhlemann, Y.; Weiss, D.S.; Weinrauch, Y.; Zychlinsky, A. Neutrophil extracellular traps kill bacteria. Science 2004, 303, 1532-1535. [CrossRef] [PubMed]

3. Schonrich, G.; Raftery, M.J. Neutrophil extracellular traps go viral. Front. Immunol. 2016, 7, 366. [CrossRef] [PubMed] 
4. Narayana Moorthy, A.; Narasaraju, T.; Rai, P.; Perumalsamy, R.; Tan, K.B.; Wang, S.; Engelward, B.; Chow, V.T. In vivo and in vitro studies on the roles of neutrophil extracellular traps during secondary pneumococcal pneumonia after primary pulmonary influenza infection. Front. Immunol. 2013, 4, 56. [CrossRef] [PubMed]

5. Sumby, P.; Barbian, K.D.; Gardner, D.J.; Whitney, A.R.; Welty, D.M.; Long, R.D.; Bailey, J.R.; Parnell, M.J.; Hoe, N.P.; Adams, G.G.; et al. Extracellular deoxyribonuclease made by group a streptococcus assists pathogenesis by enhancing evasion of the innate immune response. Proc. Natl. Acad. Sci. USA 2005, 102, 1679-1684. [CrossRef] [PubMed]

6. Lavezzo, E.; Berselli, M.; Frasson, I.; Perrone, R.; Palu, G.; Brazzale, A.R.; Richter, S.N.; Toppo, S. G-quadruplex forming sequences in the genome of all known human viruses: A comprehensive guide. PLoS Comput. Biol. 2018, 14, e1006675. [CrossRef] [PubMed]

7. De Magis, A.; Manzo, S.G.; Russo, M.; Marinello, J.; Morigi, R.; Sordet, O.; Capranico, G. DNA damage and genome instability by g-quadruplex ligands are mediated by $\mathrm{r}$ loops in human cancer cells. Proc. Natl. Acad. Sci. USA 2019, 116, 816-825. [CrossRef] [PubMed]

8. Kremser, L.; Okun, V.M.; Nicodemou, A.; Blaas, D.; Kenndler, E. Binding of fluorescent dye to genomic rna inside intact human rhinovirus after viral capsid penetration investigated by capillary electrophoresis. Anal. Chem. 2004, 76, 882-887. [CrossRef] [PubMed]

9. Rankl, C.; Kienberger, F.; Wildling, L.; Wruss, J.; Gruber, H.J.; Blaas, D.; Hinterdorfer, P. Multiple receptors involved in human rhinovirus attachment to live cells. Proc. Natl. Acad. Sci. USA 2008, 105, 17778-17783. [CrossRef] [PubMed]

10. Kozma, E.; Kele, P. Fluorogenic probes for super-resolution microscopy. Org. Biomol. Chem. 2019, 17, $215-233$. [CrossRef] [PubMed]

11. Thakur, S.; Cattoni, D.I.; Nollmann, M. The fluorescence properties and binding mechanism of sytox green, a bright, low photo-damage DNA intercalating agent. Eur. Biophys. J. 2015, 44, 337-348. [CrossRef] [PubMed]

12. Friedman, R.A.; Honig, B. A free energy analysis of nucleic acid base stacking in aqueous solution. Biophys. J. 1995, 69, 1528-1535. [CrossRef]

13. Real-Hohn, A.; Provance, D.W., Jr.; Goncalves, R.B.; Denani, C.B.; de Oliveira, A.C.; Salerno, V.P.; Oliveira Gomes, A.M. Impairing the function of mlck, myosin va or myosin vb disrupts rhinovirus b14 replication. Sci. Rep. 2017, 7, 17153. [CrossRef] [PubMed]

14. Ganjian, H.; Zietz, C.; Mechtcheriakova, D.; Blaas, D.; Fuchs, R. Icam-1 binding rhinoviruses enter hela cells via multiple pathways and travel to distinct intracellular compartments for uncoating. Viruses 2017, 9, 68. [CrossRef] [PubMed]

15. Abou Alaiwa, M.H.; Launspach, J.L.; Sheets, K.A.; Rivera, J.A.; Gansemer, N.D.; Taft, P.J.; Thorne, P.S.; Welsh, M.J.; Stoltz, D.A.; Zabner, J. Repurposing tromethamine as inhaled therapy to treat cf airway disease. JCI Insight 2016, 1, e87535. [CrossRef] [PubMed]

(C) 2020 by the authors. Licensee MDPI, Basel, Switzerland. This article is an open access article distributed under the terms and conditions of the Creative Commons Attribution (CC BY) license (http://creativecommons.org/licenses/by/4.0/). 